\title{
LITERIDAD educación inclusiva. Análisis y reflexiones en la
educación superior ecuatoriana
}

\section{Inclusive education. Analysis and reflections in Ecuadorian Higher Education}

(D) Ruth Germania Clavijo Castillo es docente investigadora de la Universidad de Cuenca (Ecuador) (ruth.clavijo@ucuenca.edu.ec) (https://orcid.org/0000-0002-3617-9626)

(iD) María José Bautista-Cerro es docente investigadora de la Universidad Nacional a Distancia (UNED) (España) (mjbautistac@edu.uned.es) (https://orcid.org/0000-0002-3658-2191)

Recibido: 2019-01-14 / Revisado: 2019-04-27 / Aceptado: 2019-11-29 / Publicado: 2020-01-01

\section{Resumen}

El acceso a una educación en igualdad de condiciones y oportunidades de aprendizaje es una aspiración y un compromiso que debe ser asumido por todos los gobiernos e instituciones públicas y privadas. A nivel internacional, y específicamente en Ecuador, se han generado leyes y acuerdos que han apoyado el proceso de educación inclusiva en todas las etapas. El propósito de este artículo es realizar una aportación sobre la situación y los retos que plantea la educación inclusiva en Ecuador, con especial incidencia en el ámbito de la educación superior. Para ello se ha recopilado y seleccionado, con criterios de relevancia, los acuerdos internacionales y la normativa nacional que afecta a la inclusión. A través de este análisis hemos podido constar el avance en el tratamiento de esta a nivel internacional y su reflejo en la normativa nacional. A pesar de ello, transformar las políticas, la cultura y las prácticas de las Universidades para atender a la diversidad sigue constituyendo un reto para todos los actores implicados. Como principales conclusiones de este trabajo se establece la necesidad de avanzar hacia un modelo de universidad basado en los principios de la inclusión educativa ya establecidos, no solo como una cuestión imprescindible para mejorar los procesos de calidad, sino como un elemento clave en orden a construir sociedades más justas, democráticas y equitativas.

Descriptores: Educación, inclusión, diversidad, normativa, universidad, sostenibilidad.

\section{Abstract}

Access to education on equal terms and with equal learning opportunities is an aspiration and a commitment that must be assumed by all governments and public and private institutions. At the international level, and specifically in Ecuador, laws and agreements have been generated that have supported the process of inclusive education at all stages. The purpose of this article is to make a contribution on the situation and challenges posed by inclusive education in Ecuador, with special incidence in the field of higher education. To this end, international agreements and national regulations affecting inclusion have been compiled and selected, with relevant criteria. Through this analysis we have been able to note the progress in the treatment of inclusion at the international level and its reflection in national legislation. In spite of this, transforming the policies, culture and practices of Universities in order to attend to diversity continues to be a challenge for all the actors involved. The main conclusions of this work are the need to move towards a university model based on the principles of educational inclusion already established, not only as an essential issue to improve quality processes, but also as a key element in order to build more just, democratic and equitable societies.

Keywords: Education, inclusion, diversity, regulations, university, sustainability.

Forma sugerida de citar: Clavijo Castillo, R. G., \& Bautista-Cerro, M. J. (2020). La educación inclusiva. Análisis y reflexiones en la educación superior ecuatoriana. Alteridad, 15(1), 113-124. https://doi.org/10.17163/alt.v15n1.2020.09 


\section{Introducción}

La exclusión se da en todos los contextos y situaciones de la vida cotidiana, en ocasiones de manera directa y perceptible y en otras de forma sutil. A veces, las situaciones donde se produce exclusión son aceptadas socialmente de manera que es difícil percibirla incluso por aquellos que la sufren. Lo cierto es que el avance de la democracia y la mayor percepción de los Derechos Humanos hacen que la inclusión se convierta en un tema relevante en todos los sectores de nuestras sociedades y especialmente el educativo.

En el ámbito educativo podemos hablar de exclusión de hecho o de derecho (Parrilla, 2002). En ocasiones puede existir una normativa que establezca quienes tienen derecho a recibir educación, pero no siempre era necesario establecerla. La exclusión se puede concretar en favorecer una educación para determinadas élites de la sociedad, quedando las grandes mayorías excluidas de los procesos educativos. Pero más allá de los motivos económicos, los fenómenos de exclusión tienen su razón de ser en la diferencia sin carácter valorativo. Se rechaza aquello que es diferente, se cierra la puerta a aquello que no encaja en los cánones de "normalidad" que se establecen en un momento determinado.

La inclusión en el ámbito educativo conlleva actitudes de profundo respeto por las diferencias y una responsabilidad para hacer de ellas una oportunidad para el desarrollo, la participación y el aprendizaje. El diseño de procesos educativos que sitúen la inclusión como eje vital requiere la participación de muchos agentes sociales. La UNESCO (2006) concibe la educación como un factor de cohesión siempre y cuando considere la diversidad de las personas y de los grupos humanos evitando ser un factor de exclusión social (Calvo \& Verdugo, 2012). La educación inclusiva se basa en un sistema de valores determinados que se acogen para poder poner en marcha el proceso de enseñar y aprender (Colmenero, 2015). Supone, por tanto, un cambio de enfoque para acoger la flexibilidad que precisa la diferencia.

El derecho a la educación es un derecho humano incuestionable en cualquier sociedad moderna. Quedó establecido en el art. 26 de la Declaración Universal de Derechos Humanos y desarrollado en multitud de documentos posteriores, como el Pacto Internacional de Derechos Económicos, Sociales y Culturales de Naciones Unidas en 1976, la Convención de los Derechos del Niño en 1990, los Objetivos de Desarrollo del Milenio en el año 2000 o los Objetivos de Desarrollo Sostenible de 2014, entre otros muchos. En la Declaración de Derechos Humanos se establece que "la educación tendrá por objeto el pleno desarrollo de la personalidad humana y el fortalecimiento del respeto a los derechos humanos y a las libertades fundamentales; favorecerá la comprensión, la tolerancia y la amistad entre todas las naciones y todos los grupos étnicos o religiosos" (Naciones Unidas, 1999) y para ello se definen cuatro características básicas: disponibilidad, accesibilidad, aceptabilidad y adaptabilidad.

Bajo estas premisas no es posible delimitar el derecho a la educación teniendo como criterio una discapacidad o la diversidad. Incluir implica eliminar, reducir todas las barreras al aprendizaje (Booth, 2000) y consecuentemente, avanzar hacia una sociedad más justa, más incluyente para todos y hacia todos. Teniendo en cuenta la diversidad presente en las sociedades la escuela y las instituciones educativas deben ser reflejo positivo de esa diversidad apoyando el trabajo de padres, docentes, directivos, nuevos profesionales y agentes sociales que siguen está misma línea (Corral, Villafuerte, \& Bravo, 2015). Pero es necesario advertir que la sola presencia física del estudiante no representa que la institución se convierta en espacio inclusivo, esto constituye apenas el inicio del proceso de educación inclusiva.

En el Ecuador en la década de los 60 y 70 si bien la educación se caracterizó por una apreciación alta de la escuela pública (Isch, 2011) podemos afirmar que se trataba de un modelo excluyente. La escuela aceptaba solo a 
los niños considerados "normales" mientras que los "diferentes" no encontraban ni el acceso ni los itinerarios necesarios según sus necesidades. La exclusión educativa se irá reduciendo al pasar los años y el diseño de las reformas instauradas por políticas sociales y educativas cuyo principal objetivo era el de transformar internamente los establecimientos educativos en el país.

Las políticas generadas a nivel internacional por organizaciones como: Oficina Regional de Educación para América Latina y el Caribe (OREALC) Organización de las Naciones Unidas para la Educación, la Ciencia y la Cultura (UNESCO), Organización de Estados Iberoamericanos (OEI), así como también a nivel nacional: Secretaría Nacional de Planificación y Desarrollo (SENPLADES), Consejo Nacional de Igualdad de Discapacidades (CONADIS) Secretaría Nacional de Educación Superior, Ciencia, Tecnología e Innovación (SENESCYT), Consejo de Educación Superior (CES) entre otras, pretenden fomentar el respeto a la diversidad como valor fundamental de la sociedad. Todo ello considerando que la única forma de transformar el sistema educativo es con la participación de docentes, estudiantes, autoridades y familias. Para Booth (2000) una educación con calidez implica transformaciones significativas al sistema educativo, "en sus culturas, en sus políticas y prácticas” (Booth, 2000, p. 11).

Las recomendaciones internacionales y las políticas públicas afines con la educación han progresado en la generación de mecanismos que permiten avanzar en la inclusión, mirando la diversidad desde un marco más comprensivo, destacando en positivo los rasgos distintivos de los estudiantes: diferencias de raza, etnia, género, lengua, religión, estilos de aprender, condición personal, capacidades artísticas, manuales e intelectuales diferentes (Muñoz, 2009; Herdoíza, 2015; Gallegos, 2015). Desde esta óptica, se pretende determinar el impacto de la normativa en torno a la inclusión educativa, normativa que garantiza una educación enfocada hacia el respeto de las individualidades y la diversidad.

\section{Sobre la educación inclusiva}

Coincidimos con Booth cuando afirma que "todos los seres humanos somos diferentes, no hay dos seres idénticos. Estas diferencias individuales se deben reconocer en los procesos educativos" (Booth, 2000 p. 9).

Desde la negación del derecho de diversos grupos humanos a la educación, mujeres, individuos con discapacidad, personas de culturas distintas, niñas y niños, hijos de desplazados o migrantes, hasta la situación actual de admisión parcial o completa en los diferentes niveles del sistema educativo se ha transitado un camino extenso. "El trayecto ni ha sido único (de hecho, podemos hablar de distintos caminos y rutas hacia la inclusión) ni lineal (se ha desarrollado a ritmos y tiempos distintos según colectivos y países)" (Parrilla, 2002, p. 11).

La inclusión tampoco ha tenido una concepción unívoca en sus referentes, más bien ha evolucionado con sentidos y marcos teóricos diferentes. En principio surge marcada por una nueva conciencia social, que la UNESCO inicia y promueve, sobre las desigualdades en el tratado de los derechos humanos, prestando atención especial sobre las diferencias en el respeto de la educación como un derecho. En este sentido, la educación inclusiva queda entendida como un proceso dinámico, abierto y flexible que reconoce y aprecia la diversidad en los niños, niñas y adultos sin hacer distinciones de ningún tipo. Incluir significa poner en marcha un sistema educativo basado en la diversidad, equidad y participación en un entorno ordinario (Calvo \& Verdugo, 2012; Echeita \& Navarro, 2014; Colmenero, 2015; Fernández \& Pérez, 2016). Para la UNESCO (2004) la inclusión educativa significa desarrollar escuelas que atiendan a todos y todas, cualesquiera sean sus características o dificultades; por su parte Booth y Ainscow (2002) la conciben como un conjunto de procesos que pretenden eliminar o disminuir las barreras que no permiten la participación de todos. 
En el ámbito educativo todos los ciudadanos deben tener garantizada una educación de calidad. Las instituciones educativas son responsables de hacer real el derecho a una educación que despliegue al máximo en potencial de cada persona. Considerando a la diversidad a partir de un enfoque más comprensivo, enfatizando que el estudiantado forma parte de una aglomeración compuesta por diferencias (Booth, 2000; Muñoz, 2009; Echeita \& Navarro, 2014). En este contexto las políticas públicas vinculadas con la educación han progresado en la generación de mecanismos que favorecen la inclusión educativa, en base a que el futuro de la sociedad es fruto de su educación, por lo que la sociedad y las instituciones educativas hemos de apostar por ofrecer una educación de calidad (Colmenero, 2015; Herdoíza, 2015).

\subsection{Acuerdos Internacionales}

La cultura humana ha desarrollado acuerdos y compromisos internacionales que empujan la inclusión educativa, reconociendo el derecho de educarse en la diversidad con equidad y calidad. A continuación, resumimos estos grandes momentos:

- El derecho a la educación queda señalado, en el Artículo 26 de la Declaración Universal de Derechos Humanos (1948), donde se reconoce el valor humano de la educación, fundamentado en sólidos cimientos no solo morales sino también jurídicos. La educación es una herramienta indudable para hacer efectivos, defender y apadrinar otros derechos humanos. El ejercicio de la libertad, disfrutar de la seguridad y de un medio ambiente adecuado se ven fortalecidas con una educación de calidad. El reconocimiento del valor humano intrínseco de la educación se ha consolidado en grandes convenciones que hacen hincapié en la importancia de la inclusión plena de las personas con diversidad (Valenciano, 2009).
- En el año 1975 a través de la Declaración de los derechos de los impedidos efectuada por la Asamblea General de la ONU se establecen normas sobre igualdad de oportunidades para aquellas personas que tienen discapacidad. Se consolida el reconocimiento de los derechos a todos, exhortando a la comunidad a no ejercer distinción fundamentada en la raza, sexo, idioma, color, religión, credos, origen, condición socioeconómica, nacimiento o cualquier otra característica (Parra, 2010). Para Muñoz (2009), a través de esta declaración se promueve los cambios de políticas para asistir la educación integrada de los estudiantes con discapacidad en las escuelas regulares. Esta declaración que intenta otorgar derechos básicos a las personas con discapacidad, su mayor interés radica en prevenir las discapacidades y estimular la rehabilitación (Parra, 2010).

- El informe Warnock, elaborado por Mary Warnock, para Inglaterra, Escocia y Gales publicado en 1978, indica que la educación es un bien, que debe ser reconocido como un derecho de todos, y que las necesidades educativas (NEE) son habituales a todos los estudiantes, las que requieren de atención y recursos específicos distintos (Warnock, 1990).

- La Declaración Universal de los Derechos del Niño 1959: Establece la igualdad de derechos para todos, niñas y niños, reconocidos como persona, sin ningún tipo de excepción, con sus intereses y necesidades (Naciones Unidas, 1959).

- Conferencia de Jomtien, Declaración Mundial de Educación para Todos, 1990, en la misma se estableció el objetivo de "educación para todos", con la finalidad de motivar la igualdad y el acceso universal a la educación, asumiendo a la atención a la diversidad como un valor y como potencial para el progreso de la sociedad (UNESCO, 1990). 
- Conferencia mundial sobre Necesidades Educativas Especiales: Acceso y Calidad. Salamanca, 1994: Establece que las escuelas deben acoger a todos los niños, sin observar sus condiciones personales, culturales o sociales, que es emergente impartir enseñanza a todos los estudiantes dentro del sistema regular de educación y se sostiene que las aulas escolares constituyen la medida más poderosa para combatir las actitudes segregacionistas, esto permite crear comunidades de acogida, que posibilite la construcción de una sociedad con educación para todos (Naciones Unidas, 1994).

- Foro consultivo internacional de Educación para todos 2000, celebrado en Dakar, en este espacio se reafirma la necesidad de prestar atención sobre el acceso a la educación y la inclusión de los estudiantes históricamente marginados y desaventajados. Para alcanzar esta meta los sistemas educativos deberán ser integrales, considerando sobre todo las necesidades básicas de aprendizaje y promoviendo la equidad entre todos los seres humanos (OEI, 2000).

- En diciembre de 2006, la Asamblea General en su resolución 61/106 aprueba la Convención sobre los derechos de las personas con discapacidad y su protocolo Facultativo. Dicho protocolo constituye un tratado internacional donde se reconocen derechos y obligaciones que tienen los Estados partes para promover, proteger y asegurar su cumplimiento. De igual manera se reconoce la igualdad de todos los derechos humanos y libertades fundamentales que debe ser garantizadas a todas las personas, mediante un sistema de educación inclusivo en todos los niveles (Naciones Unidas, 2008).

- Más recientemente y aunque con carácter general la Agenda 2030 sitúa la educación como motor de todos los Objetivos de Desarrollo Sostenible (ODS) y enuncia un objetivo, el número 4, que se centra especí- ficamente en la educación "Garantizar una educación inclusiva, equitativa y de calidad y promover oportunidades de aprendizaje durante toda la vida para todos" (Naciones Unidas, 2015). Este objetivo queda desarrollado en metas concretas que deben alcanzar todos los países y en los que la inclusión es un vector esencial.

Estas conferencias y acuerdos internacionales posibilitan que la educación quede configurada como un continuo esfuerzo para responder a la diversidad. Así la educación ha pasado de ser un privilegio de pocos a ser un derecho de todos (Clavijo, López, Mora, Ortiz \& Cedillo, 2016).

En el caso de América Latina y el Caribe aún hace falta superar una serie de "brechas estructurales" como la desigualdad de género, de raza, etnia y población, la pobreza que persiste actualmente, la casi nula efectividad de las políticas públicas de inclusión social, la escasa calidad de los servicios de salud y educación, (...) (Naciones Unidas, 2015), desigualdad que supone un esfuerzo adicional para que la educación inclusiva sea una realidad en todos los niveles del sistema educativo, sobre todo en la educación superior como un mecanismo que permite corregir las desigualdades sociales (De la Cruz, 2012; Herdoíza, 2015; Vessuri, 2016; Fernández \& Pérez, 2016).

\subsection{La educación inclusiva en el país. Base legal}

Si bien la educación inclusiva en otros países de Latinoamérica como México, Brasil, Chile ya tiene un camino recorrido, en Ecuador se comienza a abordar a profundidad el tema a inicios de este siglo. El término educación inclusiva es de uso relativamente reciente y todavía se encuentra en proceso de consolidación, tanto interpretativa como práctica (Clavijo et al., 2016). En torno a 1940 podemos situar sus inicios y se caracterizó por un carácter asistencial, en el que primaba un enfoque médico. La responsabilidad de la atención educativa a estudiantes que no tenían cabida 
en el sistema escolar recaía sobre los progenitores y organizaciones particulares que, por iniciativa propia, crearon centros para dar atención educativa a niños y niñas con discapacidad. Esta atención se basaba en criterios de caridad y beneficencia (Vicepresidencia de la República, 2011).

En la década de los 70 podemos hablar del inicio del periodo de la institucionalización. Varios organismos públicos y privados asumen responsabilidades en los espacios de la educación, la salud y bienestar social, para afrontar y corregir necesidades de la población con discapacidad. Posteriormente se adopta un enfoque rehabilitador determinado por la adaptación de currículos para cada una de las discapacidades. Estos currículos son oficializados mediante acuerdos ministeriales y son paralelos a los de la educación regular, aunque las instituciones educativas trabajan de modo aislado y de forma diferente a la educación regular (Vicepresidencia de la República, 2011).

Acciones importantes se desarrollan en las décadas de los 60 a los 80 como la publicación de Reglamentos (Ley Orgánica de Educación, Ley de Educación y Cultura del 77, Ley y Reglamento de Educación Especial) acuerdos ministeriales y normativa que regulan la Educación Especial. Toda esta base legal presenta una orientación inclusiva y por primera vez "aparece el término de educación inclusiva, pero mezclado con el de integración" (Rosano, 2008, p. 60); pese a esta mezcla que en principio hace ver que la educación sigue incrustada en la integración educativa, el hablar de inclusión permite al país caminar con pasos sólidos hacia la educación inclusiva.

Los eventos mundiales que se producen en la década de los 90 (Declaración Mundial de Educación para Todos, Declaración de los Impedidos, Foro Mundial de Educación, entre otros) empujan también a Ecuador a direccionar la educación desde la perspectiva inclusiva. Se generan medidas para que los estudiantes sean atendidos con las mismas oportunidades. Se rompe la idea en general aceptada de que solamente los estudiantes con NEE necesitan apoyo, reconociendo que cualquier educando puede necesitar apoyo para acceder al currículo oficial.

En el año 2003 entra en vigor el Código de la Niñez y de la Adolescencia en el que se sostiene que, ante la ley, todas las niñas, niños $\mathrm{y}$ adolescentes son iguales independientemente de su condición (Congreso Nacional, 2003). Evidenciándose la voluntad del Estado ecuatoriano para favorecer la inclusión en las instituciones educativas adoptando la diversidad como un elemento positivo. Más adelante con la coyuntura política de la época, se aprueba en el 2006, mediante consulta popular a nivel nacional con más del 66\% de votación, el Plan Decenal de Educación. Las ocho políticas de este plan tienen un enfoque inclusivo y buscan garantizar la educación de todas las personas, con independencia de las situaciones personales, culturales, étnicas, sociales y de discapacidad. Las líneas presentes tendentes a universalizar la educación inicial y la educación general básica, mejorar la infraestructura, la calidad y equidad, revalorizar la profesión docente (SENPLADES, 2012), entre otras, constituye políticas que asisten a la promoción de la educación inclusiva.

En la Constitución de la República del Ecuador aprobada mediante plebiscito en el año 2008, se sitúan objetivos vinculados a mejorar progresivamente la calidad educativa, basada en un enfoque de derechos, de género, intercultural e inclusiva, buscando robustecer la unidad en la diversidad y sobre todo garantizando la permanencia y la culminación de los estudios, así como examinar otras formas de diversidad, mediante el análisis de la capacidad de cada una de ellas en su aporte para construir relaciones de convivencia, equidad, diálogo y creatividad (Constitución, 2008). La educación queda plasmada como un derecho de las personas a lo largo de su vida y como un deber que el Estado ecuatoriano no puede prescindir, ni excusar. Constituye un área prioritaria de la política pública.

La educación se centrará en el ser humano y garantizará su desarrollo holístico, en el marco del respeto a los derechos humanos, al medio 
ambiente sustentable y a la democracia; será participativa, obligatoria, intercultural, democrática, incluyente y diversa, de calidad y calidez; impulsará la equidad de género, la justicia, la solidaridad y la paz. (Constitución, 2008, p.16)

En esta misma línea, con la promulgación de la Ley de Educación Intercultural (LOEI, 2011), se hacen efectivos los derechos constitucionales que poseen las personas con discapacidad, garantizando la inclusión a las instituciones educativas, eliminando las barreras para el aprendizaje. Se reconoce como titulares del derecho a la educación de calidad, laica, libre y gratuita en los niveles inicial, básico y bachillerato, así como a una educación permanente a lo largo de la vida, a todos los y las habitantes del Ecuador (...). A recibir una formación integral y científica, que asista al desarrollo pleno de su personalidad, capacidades y potencialidades, considerando sus derechos, libertades fundamentales, a través de la promoción de la igualdad de género, la no discriminación, el reconocimiento de las diversidades, la participación, la libertad y cooperación. Mediante esta ley se promueve que cada uno sea "tratado con justicia, dignidad, sin discriminación, con respeto a su diversidad individual, cultural, sexual y lingüística, a sus convicciones ideológicas, políticas y religiosas, (...)" (Legislación, 2011, p.18).

La LOEI promueve equidad e inclusión, garantiza a todas las personas el acceso, permanencia y culminación en el Sistema Educativo. Promueve políticas inclusivas, mediante la creación de medidas de acción afirmativa y una cultura escolar incluyente, motivando la igualdad de oportunidades a comunidades, pueblos, nacionalidades y grupos (Legislación, 2011).

Tiempo después se aprueba la Ley de Discapacidades (2012); con ello el Ministerio de Educación debe asumir algunas responsabilidades permitiendo que, niños y jóvenes con discapacidad se integren a la educación regular. Un aspecto clave en esta ley, es el reconocimiento del derecho a la educación, estableciendo que el Estado gestione y garantice que aquellas personas que tengan discapacidad accedan, permanezcan y culminen el Sistema Nacional de Educación y el Sistema de Educación Superior (CONADIS, 2012).

Paralelamente aparece el Plan Nacional del Buen Vivir (PNBV) el mismo que contempla sugerencias desde la política pública para desarrollar componentes de inclusión, protección social, integración y territorio. A partir de este plan se fortalece la idea que el sistema educativo, identifica y valora a todas las personas, en especial a los grupos de atención prioritaria y se reconoce la pluralidad de comunidades, pueblos y nacionalidades (SENPLADES, 2012). El PNBV comprende el saber, mediante el reconocimiento de las diversidades en igualdad de oportunidades, los signos de conducta para la interacción social, así como su relación con la naturaleza, los derechos fundamentales, el diálogo intercultural entre otros (SENPLADES, 2012). Ante esta nueva filosofía constituye política de estado legitimar y respetar las diversidades socioculturales eliminando toda forma de discriminación. Acciones básicas que deben ser promovidas desde todas las instituciones educativas y sobre todo fortalecerse desde las Instituciones de Educación Superior (IES) (Herdoíza, 2015).

En el Plan Toda una Vida (2017-2013), que especifica la política pública del territorio ecuatoriano, se establece como responsabilidad del Estado proveer una educación pública, gratuita y universal; replantear la educación superior como bien público y motor de desarrollo. La educación superior constituye un espacio para lo formación integral e incluyente de todos por lo que, el reto es mejorar la calidad, así como el acceso e incremento significativo de la cobertura (SENPLADES, 2017).

\section{La educación inclusiva en la Educación Superior}

Por lo que respecta a la Educación Superior, en el 2010 se promulga la Ley de Educación Superior (LOES). El objetivo de la ley radica en definir sus 
principios y garantizar el derecho a la educación superior de calidad que apunte a la excelencia, a través del acceso universal, permanencia, movilidad y egreso sin diferencia alguna. Se sustenta en el principio de igualdad de oportunidades buscando garantizar a todos, iguales posibilidades, sin diferencias de género, orientación sexual, creencias, etnia, cultura, convicción política, condición socioeconómica o discapacidad (Asamblea Nacional, 2010).

En este contexto, en consonancia con la normativa señalada, superar las desigualdades y la exclusión son quizás los retos más apremiantes de estos tiempos para los países latinoamericanos $y$ del mundo en general. Son las IES, en este escenario de globalización las invitadas a contribuir en la transformación de la sociedad, mediante la formación de profesionales con conocimientos y capacidades que les permita brindar respuestas a las necesidades del desarrollo social y ayuden a construir una ciudadanía preparada para la convivencia humana y su bienestar (Asamblea Nacional, 2010; Fernández \& Pérez, 2016).

Situar la inclusión como eje fundamental de la educación especialmente en el sistema universitario supone favorecer sociedades más equitativas y justas. Como señala el PNUD en su web: "El objetivo de lograr una educación inclusiva y de calidad para todos se basa en la firme convicción de que la educación es uno de los motores más poderosos y probados para garantizar el desarrollo sostenible". La formación en cualquier actividad profesional busca contribuir a conocer y promover los Derechos Humanos, así como también a conocer los principios democráticos, y de igualdad entre mujeres y hombres, la solidaridad, la protección medioambiental, el acceso universal y fomentar una cultura de paz (Naciones Unidas, 2015).

Si bien la educación inclusiva debe ser asumida de forma individual, las IES ecuatorianas desplegaran acciones que se traduzcan hacia lo colectivo (Asamblea Nacional, 2010; Herdoíza, 2015); todas las carreras deberán asegurarse de que sus nuevos profesionales no queden al mar- gen de la acción social para garantizar el buen vivir que plantea la Constitución. Los profesores ayudarán en la formación de competencias que permita encontrar soluciones a problemas específicos de su carrera, asumiendo estilos de vida que reconozca y respete la diversidad. Existen algunos estudios como el de Rodríguez (2004), Espinosa, Gómez, y Cañedo (2012) o Fajardo (2017), que señalan a las universidades ecuatorianas como instituciones responsables en la formación de comportamientos personales y sociales, que salvaguarden la dignidad y paridad de todos los estudiantes, reclamando derechos inalienables desde el reconocimiento y respeto de su diversidad, trabajando valores como la no violencia, la promoción de la salud, valores ineludibles para el desarrollo educativo inclusivo. En coherencia con estos principios, la Universidad de Cuenca, alineada dentro de la Constitución de la República, los OBV y el Plan Toda una Vida, cuenta con políticas institucionales de acción afirmativa como políticas y prácticas encaminadas a eliminar todo tipo de discriminación, garantizando la igualdad de oportunidades de todos los grupos humanos, para ello en la actualidad desde el Departamento de Bienestar Universitario se trabaja con el "Instructivo que regula el proceso de inclusión educativa en la Universidad de Cuenca" (2017) buscando asegurar la educación superior como un derecho de todos. El trabajo en atención a la diversidad y el Instructivo indicado pretenden sensibilizar e involucrar a todos los integrantes de la comunidad universitaria, a reconocer la diversidad, eliminando barreras que limitan el aprendizaje y garantizar el ingreso, la permanencia y culminación de sus estudios a todos los estudiantes que ingresan a la Universidad (Universidad de Cuenca, 2017).

Como puede apreciarse, la educación inclusiva es un referente básico en la ordenación reglamentaria del sistema educativo de nuestro país, vinculándose al reconocimiento de la dignidad e igualdad de las personas como parte de sus derechos que son inalienables, se orienta en com- 
prometer la acción universitaria en la superación de las diferencias mediante la compensación de las distintas desigualdades (SENPLADES, 2017), estos aspectos contemplados en el Plan Toda una Vida, buscan que nuestras universidades contribuyan a conformar una sociedad hermanada, basada en el reconocimiento y respeto de la diversidad humana en todas sus expresiones, mediante un marco habitual de derechos y deberes compartidos, una sociedad que se esfuerce por equiparar las oportunidades de todos, con particular consideración en aquellos que por distintas razones se encuentren en condiciones de mayor vulnerabilidad (Herdoíza, 2015).

\section{Retos y oportunidades en la educación inclusiva}

La normativa sobre inclusión en el país pretende promover el verdadero sentido de la educación inclusiva, entendida como derecho fundamental y como elemento que determina la calidad educativa. El camino ya se ha iniciado, los países de América Latina y el Caribe en los últimos años han efectuado importantes avances en el desarrollo de la educación, extendiendo la durabilidad de la educación básica (obligatoria); ampliando la cobertura en los diferentes niveles; diseñando currículos que den respuesta a la diversidad; mejorando la dotación de materiales $y$ de infraestructura y sobre todo ejecutando distintas acciones que contribuyan a la formación docente (Vessuri, 2016). Más allá de las acciones gubernamentales, la responsabilidad de las IES supone contestar con nuevos planteamientos y respuestas apropiadas que permita confrontar los desafíos de la diversidad, desde el accionar de las instituciones como de sus profesionales (Parrilla, 2002). Facilitar el acceso a una IES implica que profesores, estudiantes, autoridades y demás personal de la institución implementen acciones institucionales para atender con calidad y responsabilidad a toda la población sobre todo aquellos que presentan diversidad (Blanco \& Duck, 2010). Más allá de las cuestio- nes administrativas, la educación inclusiva debe luchar contra determinadas actitudes de todos los involucrados en el proceso educativo. Se trata de aplicar en la universidad pública, de manera particular, los postulados de la cultura de la diversidad (Juárez, Comboni \& Garnique, 2010).

Teniendo en cuenta lo dicho anteriormente, y en consonancia con las aportaciones internacionales y la legislación ecuatoriana, consideramos una serie de acciones a desarrollar en las IES, principalmente en tres frentes:

- Institucional: Crear las condiciones físicas, materiales y estructurales para garantizar el acceso y la permanencia de los estudiantes independientemente de sus características. Esto implica establecer y mantener infraestructuras accesibles que eliminen barreras físicas que restringen la participación en los procesos de aprendizaje.

- Además, es conveniente facilitar apoyo económico para estudiantes con diversidad con la finalidad de garantizar la culminación de sus estudios universitarios. Así también implementar recursos humanos de soporte al Departamento de Bienestar Universitario, para apoyar la generación una cultura inclusiva.

- Formativo: Por lo que respecta a los docentes, establecer una formación específica y el apoyo necesario para mejorar la práctica educativa. Se busca con ello no solo la sensibilización individual sino, además, dotar a los docentes de herramientas para trabajar una educación inclusiva de calidad.

- Específicamente es importante diseñar e implementar Maestrías de Educación Inclusiva que permita fomentar el respeto y valoración de la diversidad en la comunidad universitaria mediante la formación en educación inclusiva

- Investigador: implementar y favorecer líneas de investigación sobre Educación Inclusiva: políticas, culturas y prácticas inclusivas que posibiliten perfeccionar la 
práctica docente, proporcionando de esta manera respuesta a la diversidad de los estudiantes.

- La responsabilidad de hacer posible una educación verdaderamente incluyente debe contar con el compromiso de todos y no recaer únicamente en las instituciones educativas. Dotar de recursos es una responsabilidad que ningún gobierno debe eludir.

\section{Conclusiones}

En los últimos años la normativa creada, reconoce la necesidad de formar en educación inclusiva en los contextos universitarios. Frente a la diversidad social el reto es conseguir una educación inclusiva, de calidad, para todos, por tanto, la universidad deberá responder está demanda social incorporándola en sus estructuras y currículos.

La inclusión efectiva pasa primeramente cuando se democratiza el acceso al conocimiento, cuando además se facilita la igualdad de oportunidades que viabilice el desarrollo de las competencias requeridas para actuar en la sociedad aumentando las posibilidades de las personas para elegir; incluir es mucho más que acceder a la educación, implica la posibilidad real de concluir la misma, no puede haber educación inclusiva si no empezamos a cumplir, al menos, la legislación vigente.

Una educación universitaria de calidad observará la eficacia y la eficiencia como aspectos fundamentales de las políticas formativas intentando asegurar el derecho a la educación. Necesitamos generar IES que aprueben y valoren la diferencia, reconociendo la equidad y la igualdad de oportunidades. En la práctica todos los implicados en educación superior debemos defender este verdadero sentido de la educación de calidad, trabajando desde las aulas universitarias mediante la formación de profesionales con un currículo en el que se transversalice el conocimiento de la diversidad en cada malla curricular en todas las carreras y programas académicos.
La educación constituye un derecho humano fundamental, y es imprescindible para el progreso. Permite que todos los seres humanos vivan una existencia sana, creativa y plena de sentido. Es un catalizador que busca consolidar sociedades inclusivas desde los espacios universitarios, no solo declarándola valiosa en los documentos institucionales, sino formando profesionales con capacidad para tomar decisiones y enfrentar la complejidad de la vida en la sociedad del siglo XXI.

\section{Referencias bibliográficas}

Asamblea Nacional (2010). Ley Orgánica de Educación Superior LOES. Registro Oficial Suplemento 298. Quito. 1-69.

Blanco, R., \& Duck, C. (2010). Educación inclusiva en América Latina y el Caribe. Ediciones Universidad de Salamanca. 17, 37-55.

Booth, T., \& Ainscow, M. (2002). Índice de Inclusión. Promoviendo el aprendizaje y la participación en las escuelas. UNESCO: CSIE.

Booth, T. (2000). Guía para la evaluación y mejora de la educación inclusiva. Consorcio Universitario para la Educación Inclusiva, 1-13.

Calvo, M., \& Verdugo, M. (2012). Educación inclusiva, ¿una realidad o un ideal? Edetania, 41, 17-30, ISSN:0214-8560

Clavijo, R., López, C., Mora, C., Ortiz, W., \& Cedillo, C. (2016). Educación Inclusiva en Cuenca: actitudes, prácticas y conocimientos docentes. Cuenca: Universidad de Cuenca.

Colmenero, M. (2015). Caminando hacia una educación inclusiva. Caminando hacia una sociedad inclusiva. Iniciación a la investigación, 6(4) ,1-18.

CONADIS (2012). Ley Orgánica de Discapacidades. Quito: CONADIS.

Congreso Nacional (2003). Código de la niñez y adolescencia. Quito: Registro Oficial.

Constitución (2008). Constitución de la República del Ecuador. 2008. Quito: Corporación de Estudios y publicaciones.

Corral, K., Villafuerte, J., \& Bravo, S. (2015). Realidad y perspectiva de la Educación Inclusiva de Ecuador. Investigação Qualitativa em Educação, (2), 582-587. 
De la Cruz, G. (2012). Inclusión en educación superior: de la atención a la diversidad al facultamiento del estudiantado. Revista científica electrónica de Educación y Comunicación en la Sociedad del Conocimiento, 12(2), 216-230. Publicación en línea (Semestral) Granada (España). ISSN: 1695324X.

Echeita, G., \& Navarro D. (2014). Educación inclusiva y desarrollo sostenible. Una llamada urgente a pensarlas juntas. Edetania, 46, 141-161. ISSN: 0214-8560.

Espinosa, C., Gómez, V., \& Cañedo, C. (2012). ¿Integración o inclusión? La educación superior ecuatoriana y el pleno acceso de estudiantes con discapacidad. Revista Ciencia y Sociedad, 37(3), 1-19.

Fajardo, S. (2017). La Educación Superior Inclusiva en algunos países de Latinoamérica: avances, obstáculos y retos. Revista Latinoamericana de Educación Inclusiva, 11(1), 171-197. ISSN: 07187378.

Fernández, N., \& Pérez, C. (2016). La educación superior latinoamericana en el inicio del nuevo siglo. Situación, principales problemas y perspectivas futuras. Revista Española de Educación Comparada, 27, 123-148. https://doi.org/10.5944/reec.27.2016.15044

Gallegos, M. (2011). La inclusión educativa en la Universidad Politécnica Salesiana. Alteridad, Revista de Educación, 6(2), 118-126.

Herdoíza, M. (2015). Construyendo igualdad en la Educación Superior. Fundamentación y lineamientos para transversalizar los ejes de igualdad y ambiente. SENESCYT. UNESCO.

Isch, E. (2011). Las actuales propuestas y desafíos en educación: el caso ecuatoriano. Educación Social Campiñas, 32(115), 373-391.

Juárez, J., Comboni, S., \& Garnique, F. (2010). De la educación especial a la educación inclusiva. Argumentos UAM-X. 62, 41-83.

Legislación (2011). Ley Orgánica de educación intercultural (LOEI). Quito: Corporación de estudios y Publicaciones.

Muñoz, X. (2009). Educación inclusiva: ¿Mito o realidad? Fondo de Investigación y Desarrollo en educación. Proyecto FONIDE, 1-70.

Naciones Unidas (1959). Declaración de los derechos del Niño. Asamblea General de las Naciones Unidas. Resolución 1386.
Naciones Unidas (1999). Comentario General núm. 13 al art. 13 (sobre el derecho a la educación) del Convenio internacional sobre derechos económicos, sociales y culturales (ICESCR), adoptado por el Comité de Derechos Económicos, Sociales y Culturales (CESCR). (https://goo.gl/xSq2TJ).

Naciones Unidas (1994). Declaración de Salamanca y Marco de Acción para las Necesidades Educativas Especiales. Organización de las Naciones Unidas para la Educación, la Ciencia y la Cultura. Salamanca. 1-49.

Naciones Unidas (2008). Convención sobre los derechos de las personas con discapacidad y su protocolo Facultativo. Oficina del Alto Comisionado para los Derechos Humanos. 1-64.

Naciones Unidas (2015). Transformar nuestro mundo: la Agenda 2030 para el Desarrollo Sostenible. Asamblea General. 1-40.

Organización de Estados Iberoamericanos (2000). Foro consultivo internacional sobre educación para todos. Declaración Mundial sobre educación para todos "Satisfacción de las necesidades básicas de aprendizaje" Organización para la Educación la Ciencia y la Cultura. 1-9.

Parra, C. (2010). Convención sobre los Derechos de las Personas con Discapacidad: antecedentes y sus nuevos enfoques, 16 International Law. Revista Colombiana de Derecho Internacional, 347-380.

Parrilla, Á. (2002). A cerca del origen y sentido de la Educación Inclusiva. Revista de educación, (327),11-29. Universidad de Sevilla,

Rodríguez, N. (2004). Integración de las personas con discapacidad en la educación superior en Ecuador. (http://goo.gl/vaGjEm).

Rosano, S. (2008). El camino de la inclusión educativa en punta hacienda. (Tesis maestría). Cuenca: Universidad de Andalucía.

SENPLADES (2012). Plan Nacional de Desarrollo Plan Nacional para el Buen Vivir 2009-2013: Construyendo un Estado Plurinacional e Intercultural ISBN: 978-9978-92-7946 Secretaría Nacional de Planificación y Desarrollo-SENPLADES. República del Ecuador. Quito, Ecuador. 
SENPLADES (2017). Plan Nacional de desarrollo 2017-2021. “Toda una vida”. Secretaría Nacional de Planificación y desarrollo. Quito Ecuador.

UNESCO (1990). Declaración mundial sobre educación para todos y Marco de acción para satisfacer las necesidades básicas de aprendizaje. Secretaría del Foro Consultivo Internacional sobre educación para Todos.1-42.

UNESCO (2015). El desarrollo sostenible comienza por la educación. Cómo contribuir la educación a los objetivos propuestos para después de 2015. Organización de las Naciones Unidas para la Educación, la Ciencia y la Cultura. 1-20.

UNESCO (2016). Educación 2030. Declaración de Incheon y Marco de Acción. Hacia una educación inclusiva y equitativa de calidad y un aprendizaje a lo largo de la vida para todos. Organización de las Naciones Unidas para la Educación, la Ciencia y la Cultura. 1-60.

Universidad de Cuenca (2017). Instructivo que regula el proceso de Inclusión en la Universidad de Cuenca. Unidad de Bienestar Universitario. 1-4.

Valenciano, G. (2009). Construyendo un concepto de educación inclusiva: Una experiencia compartida. Colección Investigación Salamanca. 13-24.

Vessuri, H. (2016). La ciencia para el desarrollo sostenible (Agenda 2030). Organización de las Naciones Unidas para la Educación, la Ciencia y la Cultura. UNESCO 1-19.

Vicepresidencia de la República (2011). Módulo I. Educación inclusiva y especial. Quito: Ecuador.

Warnock, M. (1990). Informe sobre NEE. Siglo Cero, 130, Madrid. 12-24. 